\title{
Predictive ManufaCtUring Systems in Industry 4.0: TRENDS, BENEFITS AND CHALLENGES
}

\author{
Bojana Nikolic, Jelena Ignjatic, Nikola Suzic, \\ Branislav Stevanov, Aleksandar Rikalovic
}
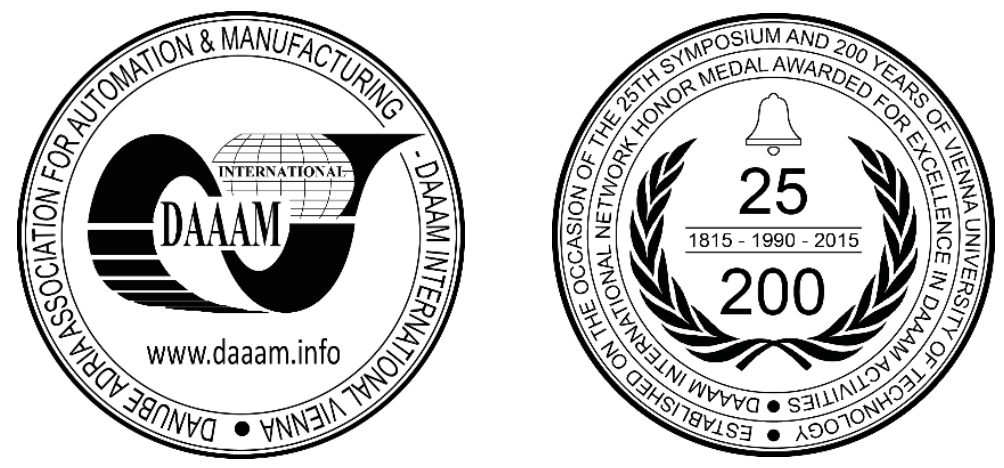

This Publication has to be referred as: Nikolic, B[ojana]; Ignjatic, J[elena]; Suzic, N[ikola]; Stevanov, B[ranislav] \& Rikalovic, A[leksandar] (2017). Predictive Manufacturing Systems in Industry 4.0: Trends, Benefits and Challenges, Proceedings of the 28th DAAAM International Symposium, pp.0796-0802, B. Katalinic (Ed.), Published by DAAAM International, ISBN 978-3-902734-11-2, ISSN 1726-9679, Vienna, Austria

DOI: $10.2507 / 28$ th.daaam.proceedings. 112

\begin{abstract}
The fourth industrial revolution, known as Industry 4.0, has tendency to push the boundaries of science and technology. This is especially true for the manufacturing industry. One of the biggest challenges facing the manufacturing industry today is how to make intelligent systems for production with "self-aware", "self-predict and "self-maintain" abilities. Predictive manufacturing systems (PMS) are new intelligent systems that provide these abilities in the production, processes and machines. The PMS combines different technologies and techniques: statistics, data mining, modelling and artificial intelligence methods. These technologies and techniques are used to convert data into information and make predictions about the observed system. This paper provides an overview of the various challenges, existing solutions and benefits of PMS, with a focus on success factors in Industry 4.0.
\end{abstract}

Keywords: Big Data; Data mining; Industry 4.0; Predictive analytics; Predictive Manufacturing Systems (PMS)

\section{Introduction}

So far, we have witnessed the development of three industrial revolutions [1]. Constant evolution of industry as well as industrial processes, is now based on cyber-physical systems (CPS), and represents an integrated computational and a physical subsystem [2]. This and the Internet of Things (IoT) leads us to think about what would be needed for the future development of manufacturing systems. The fourth industrial revolution (i.e. Industry 4.0) came as a result of the use of intelligent systems and machines that are able to predict future events and states and to suggest the optimal solutions to possible future problems [1,2,3]. In manufacturing environment, these intelligent systems are named predictive manufacturing systems (PMS).

On the one hand, PMS rely on the development of computer science, information and communication technologies, statistics, modelling and simulation [4]. On the other hand, PMS depend on the development of manufacturing science and technology [4]. PMS is an insufficiently researched emerging field that deals with issues of uncertainty and invisibles related to productivity, efficiency, flexibility and safety [5,6]. The main goal of PMS is to create manufacturing environment that enables the implementation of all new technologies based on capabilities such as "self-awareness", 
"self-predicting", "self-maintaining" and "self-learning". According to Lee et al. [5] those capabilities allow that predictive manufacturing systems have a greater transparency in order to avoid the potential issues inside the system.

Transparency is the ability of a system to eliminate and quantify the uncertainties in order to determine an objective estimation of its manufacturing capability [6]. In order to achieve transparency, manufacturing enterprise has to utilize different advanced predictive analytics along with IoT, data mining, intelligent sensor networks and big data to convert a large amount of collected data into useful information and to store it in the cloud, where it can be available at anytime and anywhere. According to [5,6,7], this represents the key effort in order to discover the invisible uncertainties in the manufacturing systems.

The trends in predictive manufacturing systems which are related with development of new technologies may vary in different geographic areas. The results and benefits achieved by implementation of PMS, are already noticeable [7] and reflected in increasing increased efficiency, enabling the transparency of the entire manufacturing system, thus reducing the production time and costs. Also, there are challenges that engineers and computer scientists are faced with, such as a large amount of generated data and the proper way of understanding, using and storing it.

The objective of this paper is to review trends, benefits and challenges for predictive manufacturing systems in Industry 4.0 environment. This paper gives the preliminary literature review of conference papers and journal articles in order to focus on further research of PMS.

The paper is organised as follows: Section 2 provides the background for the Industry 4.0, and also presents its relation with PMS, and outlines the main technologies which are used for implementation of PMS (i.e. big data, statistics, data mining and intelligent systems); Section 3 outlines the current trends, long term benefits and disparate challenges in Industry 4.0 with focus on PMS; Section 4 presents conclusions and provides directions for the future research.

\section{Background}

The social, economic and technological changes are the cause of all of the industrial revolutions [7]. The rapid growth of technologies interconnected with electronics and internet of things enables the development of manufacturing which has led to the paradigm shift and has become a fourth industrial revolution [8]. Nowadays, Industry 4.0 became a global trend which started in Germany as a high-tech manufacturing concept [8,9].

The vision of Industry 4.0 is not limited to automation of a single manufacturing facility. Its goal is to incorporate vertical and horizontal integrations of all core functions, from manufacturing, procurement and warehousing, all the way to sales of the final product which leads to more rapid product development, customized production, improved management of manufacturing environments, faster supply chains, and so on. This high level of integration allows the high level of transparency across business processes, combined with new technologies which will enable a greater efficiency inside the responsive and predictive manufacturing systems that deal with various challenges including digitalization, delivery and clear decision recommendations for users [10,12].

The is implementation of cyber-physical systems combined with internet of things in Industry 4.0 which can provide predictive intelligent systems, digital representation and interoperable interfaces in order to support predictive manufacturing systems and networked production environments. The production becomes flexible and intelligent embedded devices begin to work together seamlessly [10].

In order to achieve intelligent and flexible systems, a large amount of data is required. In this large amount of data, the statistics and data mining play important role. These methods and technologies combined can transform data into useful information which can be further processed.

Figure 1 presents the PMS framework with technologies that support the development of the PMS and their interconnectedness. The big data represents the main data source without which it would not be possible to find the knowledge in the data. The process of finding the knowledge in the data, extracting it and transforming it into information is presented as knowledge discovery in databases (KDD) which uses all of the data mining techniques [11]. Other techniques that have the same goal as the data mining, but with different approaches, are statistics [12] and intelligent systems [22]. The statistical approaches are based on mathematical equations, while intelligent systems are part of artificial intelligence (AI), a subfield of computer science [2]. A separate technology that uses statistical and intelligent systems techniques in order to recognize the patterns in data regardless of its type is known as pattern recognition [30], where it could be recognized the patterns of number, picture or audio. The manufacturing systems are learning by using pattern recognition technique, where machines adopt present behaviour patterns, so that they know how to behave in the future situations. In this way, the manufacturing systems become intelligent systems with the features of prediction future events in the entire manufacturing environment.

In the rest of this section, the main technologies that enable implementation of predictive manufacturing systems in the Industry 4.0 are presented, namely: big data, statistics, and data mining and intelligent systems. 


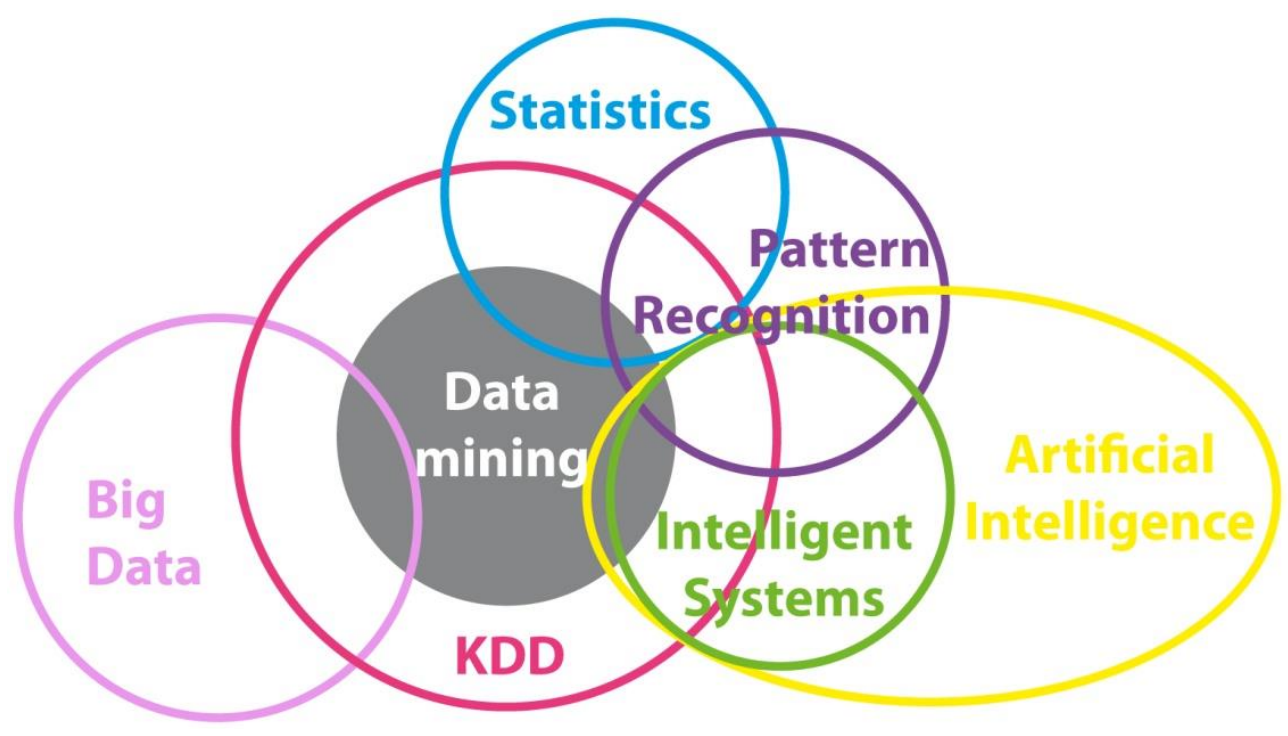

Fig. 1. The framework of predictive manufacturing systems (adopted from Srivastava, 2015 [34])

\subsection{Big data}

The Industry 4.0 includes different technologies that are joining together to discover a more effective way in order to improve manufacturing systems in every aspect of management [13]. With the implementation of new technologies in manufacturing environments, the amount of data approximately doubles every couple of years, which results in a large quantity of raw data [7]. Using the appropriate methods, algorithms and software tools, various types of data sets can be collected and extracted from the different layers in the manufacturing environment. Such extremely large and complex sets of data are named Big data $[3,13,15]$.

Today, the big data cannot be processed by using existing technologies $[15,16]$. Traditional software application processing is not capable to preserve, acquire, manage and process large amount of upcoming signals [3,14]. Technological improvements in sensing and connectivity enable seamless aggregation of raw data from various production facilities [16]. According to [5,6,14,17] the actual processing of big data into meaningful information is performed by the prognostics and health management (PHM) predictive analytics which is chosen based on the following criteria: speed of computation, investment cost and ease of deployment.

The fact is that as more and more data will be generated by ICT equipment (intelligent devices, sensors and others from the environment). Big data has ability to provide various statistical samples and results to the users. Combining the big data technology with analytics, the result is big data analytics which is able to create one of the most profound trends in manufacturing and management [16]. The big data analytics have the power to dramatically modify the current manufacturing landscape, where it could be combined with manufacturing control through the IoT, which produces many opportunities in all areas of production [10].

One of the main challenges in Industry 4.0 is how to acquire huge amounts of data from the manufacturing environments due to different technologies, machines, sensors, CPS, IoT devices and communication networks. Another challenge is transformation of collected data into information [18]. Finally, after finding a proper way of collecting all required data and transforming it into information, a challenge is how to use Big data analytics in order to discover the invisible uncertainties in the PMS [5,17].

\subsection{Statistics}

A large number of new methods (e.g. advanced statistics, machine learning, computer science and data mining) aim at taking advantage of big data [12]. Statistics has always been the most used mathematical method, that presents the traditional data analysis for gathering, reviewing, analyzing and giving conclusions from data hidden in a batch of chaotic datasets with the main goal of maximizing data value [14,15,18].

According to Hoerl, Snee, and De Veaux [12], fundamentals of statistical way of thinking is crucial for the success of big data and data mining and it is based on three fundamental principles:

- All work occurs in a system of processes that are interconnected,

- Variation exists in all processes inside the system, and

- Understanding and reducing variation is key factor to success.

These principles have been known and applied for long period of time. Nowadays, it is common thing for statistics to be replaced with more innovative methods, but advanced statistical analysis still plays a huge guidance role in making development plans in manufacturing environments in Industry 4.0. Still, advanced statistical analysis represents the basis 
for new techniques such as big data and data mining in combination with classical statistical methods such as correlation, regression, factor analysis, clustering, classification, and so on [12,13].

Correlation analysis is a statistical method for determining the law of relations and mutual restriction among observed data and according to that making predictions and giving conclusions. Also, regression analysis represents a statistical tool for revealing correlations between one variable and several others and identifies dependence relationships among variables based on a group of experiments or observed data. Factor analysis is targeted at describing the relation among many elements with only a few factors where several closely related variables are grouped into a single factor, and the few of factors are used for revealing the most information of the original data. An unsupervised study method without training data represents the cluster analysis which is used for grouping, differentiating and classifying objects according to some particular features and divide them into clusters according to these features [14]. All of these classical statistical analysis methods have one thing in common, and that is the analysis of the big data.

Big data analytics can be inferred in manufacturing environments by means of advanced statistical analysis, where the inferential advanced statistical analysis is able to make conclusions from data subject to random variations [14]. Also, it is able to make predictions based on conclusions which represent a major step forward in the implementation of predictive manufacturing systems [15].

\subsection{Data mining}

All kinds of data can be collected from the environment of PMS and transformed into valuable information which can be meaningful and useful, but the question is what the appropriate data collection technique is considering the amount and size, but also the invisibleness of raw data. The most popular technique for collecting, searching, extracting and filtering data in a systematic way is data mining [18].

Data mining is defined as a method that uses a variety of the analytical and modelling techniques for excavation of raw, but sometimes hidden and unknown data [11,13]. Also, it parses large data sets in order to find unsuspected relationships and locate latent patterns that can make accurate predictions, ensuring that the result is potentially valuable and useful information and knowledge from massive, incomplete, noisy, fuzzy and random data [15,16,19].

Data mining is used in many different areas in manufacturing and plays an important role in predictive manufacturing, where its application is most reflected in decision making and making prediction for detecting defects inside the manufacturing environments by developing the suitable software. In order to improve the efficiency and quality of software development, data mining uses different statistical techniques, such as regression, clustering, classification, but also association mining to analyse and predict defects based on collected data [12,19,20].

However, there are many challenges which need the attention of researchers. They involve industrial data such as ability to handle different types of data which can be noisy and incomplete, representation of data mining requests and results, mining at different abstraction levels of data and different sources and protection of data security [21].

\subsection{Intelligent Systems}

With the constant expansion and development of new technologies as well as an enlargement of data, the complexity of problems is increasing in industry. With that issues in mind, the intelligent systems are being investigated with the aim of finding intelligent solutions for solving complex problems [22].

The intelligent systems (IS) present systems capable providing efficient decisions making in real applications. IS are based on two major approaches - artificial intelligence and soft computing which include a variety of different techniques, such as neural networks, expert systems, fuzzy logic and fuzzy systems, genetic algorithms and genetic programming, and others. All of this techniques operate together in order to improve strategic decision making and ensure flexible processing abilities of data and information for dealing with real world situations [21,22,23,24].

In comparison with the conventional systems, the intelligent systems often provide a competitive advantage [22]. The difference between intelligent systems and conventional systems is reflected in the range of techniques and number of applications, where IS emphasize the simulation of intelligent behaviours using computing devices or software systems, and conventional systems execute the operations that people have set with the lack of any signs of intelligent behaviour $[21,25]$. Another advantage of intelligent systems over conventional systems is that IS recognizes the available data which might be incomplete, uncertain or fuzzy, where they can still make a reasonable solutions, while conventional systems can manipulate only with data which are complete or exact [26]. Moreover, automatic decision support, artificial evolution, parallel execution, virtual integration, intelligent search and optimization present unique abilities of intelligent systems which are not available in conventional information system [23,24].

The intelligent systems represent the pillar in predictive manufacturing systems in Industry 4.0. The emphasis is placed on predicting the future state of the system to reduce the unnecessary issues in early stage and tackling that issue from problem solving to problem avoidance using the machine learning techniques. With the help of adequate machine learning technique, software and tools of the intelligent systems, the PMS is able to detect disturbance and predict the occurrence of abnormality and quality of the products in the manufacturing environments where it reduces the impact of uncertainties and prevent performance loss of manufacturing systems [5,26].

Nowadays, machine learning, as a part of IS, is broadly applied in various areas of manufacturing (e.g. optimization, control, quality and troubleshooting) where its techniques are designed to extract knowledge out of existing data. The 
new knowledge supports the process of decision-making or it is used to improve the system directly. The end goal of machine learning techniques is detection of the patterns or regularities that describe the relationships and structure between the elements [27].

Different researches have different approaches to structure the field of machine learning, but the structure that is most widely used is classified by learning processes is reinforcement, supervised and unsupervised learning [28]. The reinforcement learning refers to the kind of learning where less feedback is given, because knowledgeable expert has to evaluate which of the chosen action gives the best results [27,28]. The supervised learning is machine learning where the correct response is provided by the knowledgeable expert, while the unsupervised learning is intelligent learning where evaluation of the action is not provided, because there is no knowledgeable expert [26,27]. According to Wuest et al. [27], the supervised learning is the most commonly used, because it is the best fit for most manufacturing systems due to the fact that the majority of applications can provide labelled data.

\section{Trends, benefits and challenges in predictive manufacturing systems}

As a new concept, PMS brings many opportunities and improvements inside the manufacturing environments. The conducted review led to identification of trends, benefits and challenges of PMS systems which are discussed in the following subsections.

\subsection{Trends}

The trends of PMS vary in different geographic area, but according to Liao et al. [1] and Kang et al. [3] the leading countries that are paying the most attention to new technology are Germany, USA and South Korea which allocate great resources for developing new technologies. Germany focuses on creating new values that have not been seen before and resolving various social problems by linking the things inside and outside the manufacturing environments, but also on construction of a new business models for predictive manufacturing systems [29]. In the USA the research and development programs are related to predictive manufacturing with accent on key technology assignments, including data mining, big data analytics, intelligent systems along with IoT and CPS [3]. The trends in South Korean manufacturing are similar with the trends in Germany with small change on the main manufacturing strategy from the existing assembly and device-oriented production to the innovation of development and implementation a new predictive environments [3].

The current trends in using social media networks and their development have influenced the demands and perception of consumers on product's quality, variety, innovation and speed of delivery, which lead to shorter life-cycle of a product. Such demands established the predictive manufacturing approach where the manufacturing systems in combination with corresponding software could be changed in a short period of time, which results with increase of production capacity and rapid adaption of system functionality [5]. With these demands on mind, there is no space for failure of some part inside the machine, so trends of predictive manufacturing systems are going to the next level, where they are able to predict the unsuspected events. Prediction for supply chain is also the big trend because of the possibility of prediction the need and demands of the market.

Also, according to $[5,6,15]$ there is a need for systematic integration, managing and analyzing machinery and raw data during different stages of machine life-cycle. In order to transform data into information more efficiently and to achieve better transparency of a machine's condition in the PMS, the prognostics and health management represent a critical research area that plays a huge role the development of advanced predictive tools.

\subsection{Benefits}

The technological benefits that are currently noticeable in manufacturing environments are the base of Industry 4.0. The entire present manufacturing systems is transformed into predictive manufacturing systems with the "self-aware", "self-predict", "self-maintain" and "self-learning" abilities which allow that decisions are going to be made autonomously [29]. According to Lee et al. [5] and Ferreiro et al. [30], in contrary to the conventional systems which are in use today, predictive manufacturing systems improve the performance of the equipment and strengthen the business model of industry, with the help of a set of intelligent sensing, predictive analytics and network techniques. Such an improvement of the performance leads to the prediction of the critical equipment failures, visibility of overall manufacturing process and the quality of a product, which will be guaranteed before it is produced [5].

The other advantage of the PMS is visibility of the entire manufacturing processes that leads to transparency which results the overall health condition of production systems [5]. Also, as Lee and Lapira [16] and Lee et al. [5] state that the increase of transparency in manufacturing environments has a number of benefits:

- Cost reduction - is reflected in just-in-time maintenance equipment, which maximizes the life and utilization of machine components.

- Operational efficiency - represents the knowledge about failure of the equipment that can be inferred by degradation patterns which allows better scheduling of maintenance and production. That leads to maximizing the equipment availability and uptime. Also, other associated advantages include reducing the mean time between failures and mean time to repair. 
- Product quality improvement - implies the minimization of product quality deviations in production process control, where the aim is avoidance of unnecessary rework.

PMS also have effects in the economy through cost reductions and productivity increases with constant creation of new values [3].

No one mentioned the benefits concerning the increased flexibility of the PMS in the researched papers, which allows for faster adaptation to the required changes in the entire manufacturing environments, but only as a vision of Industry 4.0 in following papers $[2,3,10,31]$.

\subsection{Challenges}

Despite the consistent development of the related technologies for the realization of PMS, there are still many issues to be considered. Transformation from present systems into more intelligent ones requires further research, development, and commercialization $[3,13]$.

In the majority of the researched papers, the first mentioned and biggest issues are related to data. According to $[13,17,29]$, the major challenge that has an effect in machine's abilities of sensoring, monitoring and gathering the big data from the manufacturing environments in order to achieve "self-aware", "self-predict", "self-maintain" and "selflearning" machines by adopting algorithms of the PHM. The development of the algorithms that are able to learn from and to handle different situations is still a great issue which can be considered as a general challenge not only for the PMS, but for entire Industry 4.0 [3,13]. Also, the collected set of data can contain an irrelevant and redundant information which can have an impact on the performance of the learning capabilities of PMS, and today's generation of PMS can only operate with continuous and nominal data values [27]. Another major concern is data security in PMS since the big data are stored in virtual cloud platforms [15]. Thus, additional researches are needed in order to improve the storage of big data.

As Liao et al. [1] and Wuest et al. [27] pointed out, the PMS represents a very complex system for managing that requires development of appropriate planning and explanatory algorithms and development of a single set of common standards which are still the big issues for the scientists. The planning and explanatory models will provide a basis for managing complex manufacturing systems, and standardisation will support a collaboration and technical description of standards. Also, standardisation can act together as a framework of standards for improving overall information exchange, sharing and integration, especially during the implementation process of Industry 4.0 [32,33].

The question about an uncertainty is still a challenge in PMS because of its invisibleness. This topic has been processed in the following papers $[5,15,26]$.

None of papers were found dealing with the issue of finding human talents for development of complex algorithms for PMS as well as other related technologies, and only one paper [10] was found that is dealing with the problems of their training for usage of intelligent devices in predictive manufacturing systems.

\section{Conclusion}

This paper provides the overview of the main technologies such as big data, statistic, data mining and intelligent systems that are used for development and implementation of predictive manufacturing systems within the Industry 4.0.

The trends are related to the development of the algorithms with accent on the key technologies. Those algorithms enable devices to work seamlessly within the PMS that will have an impact on productivity, efficiency, flexibility and safety. Other trends are related to predictive manufacturing approaches where manufacturing systems in combination with corresponding software could be changed in a short period of time. The result is production capacity incensement and functionality can adapt more rapidly.

The most challenging task is gathering the big data from the manufacturing environments in order to have enough information for developing the algorithms that are able to transform the conventional systems into the intelligent predictive systems, as well as the security of the data. Standardisation, also, represents a big issue that need to be improved in order to achieve exchange, sharing and integration of the information. Without the support of standards, the process of implementing PMS can be more difficult, which has reflections in overwhelming waste of cost, manpower and material resources.

We also found that the numerous advantages are achieved by implementation of predictive manufacturing systems such as cost reduction, operational efficiency, product quality improvement and reduction of production time. The transparency of the entire manufacturing environment represents another benefit of PMS that results an overall health of the manufacturing system.

This paper is the initial part of the research, so in future its continuity will contribute to the further exploration and development of PMS and its implementation inside intelligent manufacturing environments with focus on Industry 4.0.

\section{References}

[1] Y. Liao, F. Deschamps, E. de F. R. Loures, and L. F. Ramos. (2016). "Past, Present and Future of Industry 4.0 - A Systematic Literature Review and Research Agenda Proposal,” Int. J. Prod. Res., vol. 55, no. 12, p. 0. 
[2] M. Dopico, A. Gomez, D. De la Fuente, N. Garcia, R. Rosillo, and J. Puche. (2016). “A vision of industry 4.0 from an artificial intelligence point of view," in Proceedings on the International Conference on Artificial Intelligence (ICAI), p. 407.

[3] H. S. Kang et al. (2016). "Smart manufacturing: Past research, present findings, and future directions," Int. J. Precis. Eng. Manuf. - Green Technol., vol. 3, no. 1, pp. 111-128.

[4] L. Monostori. (2014). "Cyber-physical production systems: Roots, expectations and R\&D challenges," in Procedia CIRP, vol. 17, pp. 9-13.

[5] J. Lee, E. Lapira, S. Yang, and A. Kao. (2013). Predictive manufacturing system - Trends of next-generation production systems, vol. 46, no. 7. IFAC.

[6] J. Lee, E. Lapira, B. Bagheri, and H. an Kao. (2013). "Recent advances and trends in predictive manufacturing systems in big data environment," in Manufacturing Letters, vol. 1, no. 1, pp. 38-41.

[7] P. Tamas and B. Illes. (2017). "Process Improvement Trends for Manufacturing Systems in Industry 4.0," Acad. J. Manuf. Eng., vol. 14, no. February.

[8] J. Qin, Y. Liu, and R. Grosvenor. (2016). "A Categorical Framework of Manufacturing for Industry 4.0 and beyond," Procedia CIRP, vol. 52, pp. 173-178.

[9] S. Weyer, M. Schmitt, M. Ohmer, and D. Gorecky. (2015). "Towards industry 4.0 - Standardization as the crucial challenge for highly modular, multi-vendor production systems," IFAC-PapersOnLine, vol. 28, no. 3, pp. $579-584$.

[10] International Electrotechnical Commission. (2015). "Factory of the future $\circledR$ ".

[11] S. S. Ghuman. (2014). “A review of data mining techniques,” Ind. Manag. Data Syst., vol. 3, no. 4, pp. 1401 - 1406.

[12] R. W. Hoerl, R. D. Snee, and R. D. De Veaux. (2014). "Applying statistical thinking to 'Big Data' problems," Wiley Interdiscip. Rev. Comput. Stat., vol. 6, no. 4, pp. 222-232.

[13] J. Lee, H. A. Kao, and S. Yang. (2014). "Service innovation and smart analytics for Industry 4.0 and big data environment," in Procedia CIRP, vol. 16, pp. 3-8.

[14] M. Chen, S. Mao, and Y. Liu. (2014). "Big data: A survey," Mob. Networks Appl., vol. 19, no. 2, pp. $171-209$.

[15] N. Khan et al. (2014). "Big Data: Survey, Technologies, Opportunities, and Challenges," Sci. World J., vol. 2014, pp. 1-18.

[16] J. Lee and E. Lapira. (2013). "Predictive Factories: The Next Transformation," Manuf. Leadersh. Journal. Frost Sullivan, pp. 2-9.

[17] J. Lee, E. Lapira, S. Yang, and A. Kao. (2013). "Predictive manufacturing system - Trends of next-generation production systems," in IFAC Proceedings Volumes (IFAC-PapersOnline), vol. 46, no. 7, pp. 150-156.

[18] M. Khan, X. Wu, X. Xu, and W. Dou. (2017). "Big Data Challenges and Opportunities in the Big Data Challenges and Opportunities in the Hype," in IEEE ICC 2017 SAC Symposium Big Data Networking Track Big, no. May.

[19] M. Chandamona and A. R. Pon Periasamy. (2016). "An Improved Analysis of Data Mining Techniques on Medical Data,” Int. J. Mod. Educ. Comput. Sci., vol. 8, no. 2, pp. 42-48.

[20] A. R. Pon Periasamy and A. Mishbahulhuda. (2017). "Data Mining Techniques in Software Defect Prediction," Int. J. Adv. Res. Comput. Sci. Softw. Eng., vol. 7, no. 3, pp. 301-303.

[21] R. Ismail, Z. Othman, and A. A. Bakar. (2009). "Data Mining In Production Planning and Scheduling: A Review," in 2009 2nd Conference on Data Mining and Optimization, no. October, pp. 154-159.

[22] Z. Irani and M. M. Kamal. (2014). "Intelligent systems research in the construction industry," Expert Syst. Appl., vol. 41, no. 4 PART 1, pp. 934-950.

[23] K. A. Pupkov. (2017). "Intelligent Systems: Development and Issues," in Procedia Computer Science, vol. 103 , no. October 2016, pp. 581-583.

[24] M. K. Bharatkumar. (2015). “Intelligent System ’s Design Approaches : A Review,” Int. J. Eng. Manag. Res., vol. 5, no. 4, pp. 208-213.

[25] M. Gregor, M. Haluska, M. Fusko, and P. Grznar. (2016). “Model of Intelligent Maintenance Systems,” in 26TH DAAAM Internacional Symposium on Intelligent Manufacturing and Automation, no. 2016, pp. 1097-1101.

[26] Z. Sun. (2016). “A Framework for Developing Management Intelligent Systems,” Int. J. Syst. Serv. Eng., vol. 6, no. 1, pp. 37-53.

[27] T. Wuest, D. Weimer, C. Irgens, and K.-D. Thoben. (2016). "Machine learning in manufacturing: advantages, challenges, and applications," Prod. Manuf. Res., vol. 4, no. 1, pp. 23-45.

[28] L. Monostori. (2002). "Ai and Machine Learning Techniques for Managing Complexity, Changes and Uncertainties in Manufacturing," in IFAC Proceedings Volumes, vol. 35, no. 1, pp. 119-130.

[29] J. A. Saucedo-Martinez, M. Perez-Lara, J. A. Marmolejo-Saucedo, T. E. Salais-Fierro, and P. Vasant. (2017) "Industry 4.0 framework for management and operations: a review," J. Ambient Intell. Humaniz. Comput., pp. 113.

[30] S. Ferreiro, E. Konde, S. Fernández, and A. Prado. (2016). "Industry 4.0 : Predictive Intelligent Maintenance for Production Equipment," in European Conference of the Prognostics and Health Management Society, no. June 2016, pp. 1-8.

[31] H. Foidl and M. Felderer. (2016). "Research Challenges of Industry 4.0 for Quality Management," in Innovations in Enterprise Information Systems Management and Engineering, vol. 245, no. April, pp. 73-80.

[32] M. Freitag and M. Zelm. (2015). "Standardisation connecting the initiative 'industry 4.0' and service life cycle," CEUR Workshop Proc., vol. 1414, no. acatech 2013.

[33] L. Qing, J. Hongzhen, T. Qianlin, C. Yaotang, L. Jun, and Z. Jian. (2017). "Smart Manufacturing Standardization: Reference Model and Standards Framework,” Springer Int. Publ., vol. 10034, pp. 16-25. 